九州大学学術情報リポジトリ

Kyushu University Institutional Repository

\title{
Useful characteristics of shallow and deep marine CSEM responses inferred from 3D finite- difference modeling
}

\section{Sasaki, Yutaka}

Kyushu University, Department of Earth Resources Engineering

Meju, Max A.

Lancaster University, Department of Environmental Science

http://hdl. handle. net/2324/25555

出版情報: Geophysics. 74 (5)，pp.F67-F76，2009-08-14. Society of Exploration Geophysicists バージョン:

権利関係: (C) 2009 Society of Exploration Geophysicists. 


\title{
Useful characteristics of shallow and deep marine CSEM responses inferred from 3D finite-difference modeling
}

\author{
Yutaka Sasaki ${ }^{1}$ and Max A. Meju ${ }^{2}$
}

\begin{abstract}
Hydrocarbon reservoirs can be mapped if sufficient resistivity contrasts exist between them and their confining layers, but practical problems remain in target discrimination in deep and shallow waters, especially in the presence of heterogeneous overburden. We have developed an efficient 3D staggered-grid finite-difference controlled-source electromagnetic (CSEM) modeling code that enables study of the physics underlying some practical problems. We undertook a comparative analysis of reservoir detection in 2000-m- and 300-m-deep waters using the simulated electric and magnetic field responses of a simple 3D reservoir. We examined the effect of two types of near-surface heterogeneity (mimicking disconnected gas clouds and/or patchy geochemical alteration halos) on the 3D reservoir response. We found that small-scale, shallow heterogeneities cause distortions that are almost independent of the source frequency. These persist at all source-receiver offsets in the electric amplitude response in
\end{abstract}

deep and shallow waters and phase response in shallow water. They decrease in magnitude with increasing offset in deepwater phase response. Large-scale near-surface heterogeneities distort the horizontal electric field response more significantly than the small-scale ones, but the near-surface response gets smaller in amplitude as the offset increases. The distortions in shallow water are much smaller in magnitude than those for the deepwater case, so that the reservoir signatures still are visible on the response profiles. This might be considered as a positive feature for shallow-water inline electric field exploration. The magnetic field responses for the orthogonal direction provide diagnostic target signatures that are similar to the inline electric field responses in deep water but that are different in shallow water. The magnetic responses are affected by the airwave in a different manner from the electric field, suggesting that combined 3D electric and magnetic field analysis might be vital for handling the airwave problem.

\section{INTRODUCTION}

It is established that hydrocarbon reservoirs typically are electrically resistive and can be mapped if sufficient contrasts exist between them and the confining layers, especially in deep waters (Eidesmo et al., 2002; Ellingsrud et al., 2002; Constable and Srnka, 2007). The issues of present concern in marine exploration of hydrocarbons include shallow-water problems, background or overburden resistivity variations, formation anisotropy, rugged seabed, thin resistors of finite extent, large source-receiver (Tx-Rx) separations, poor contrast reservoirs, and the improvement of controlled-source electromagnetic (CSEM) response normalization and presentation. Exploration analysis in shallow water remains problematic because of the so-called airwave or air interaction problem (Chave and Cox, 1982; Constable and Weiss, 2006).
The effect of heterogeneous overburden on the marine CSEM response (Singer et al., 2007; Orange et al., 2009) is not well understood and might lead to erroneous interpretation or masking of potential targets as known in terrestrial magnetotellurics (e.g., Sasaki and Meju, 2006b). The seabed might be rugged in some prospects (Chandola et al., 2007; Li and Constable, 2007; Darnet et al., 2007), and blanket-type or elongate channel-fill deposits of finite extent might characterize the overburden. Such bathymetric variations and finite near-surface deposits could distort the CSEM response, and they need to be accounted for in marine CSEM simulations.

In many oil and gas fields, the reservoir is not sealed completely because of fracturing, leading to seepage especially of light hydrocarbons and the formation of shallow gas deposits (fizz gas) that cause problems for seismic reflection imaging. Conceivably, this gas

Manuscript received by the Editor 15 February 2009; revised manuscript received 12 May 2009; published online 14 August 2009

${ }^{1}$ Kyushu University, Department of Earth Resources Engineering, Fukuoka, Japan. E-mail: sasaki@ mine.kyushu-u.ac.jp.

${ }^{2}$ Formerly Lancaster University, Department of Environmental Science, Lancaster, United Kingdom; presently Petronas Research, Subsurface Technology Department, Kajang, Malaysia.E-mail: maxwell_meju@petronas.com.my.

(c) 2009 Society of Exploration Geophysicists. All rights reserved. 
seepage can result in shallow discontinuous pockets of gas that could cause distortion of the CSEM response. Seepage of gas also can lead to the formation of geochemical alteration halos in the overlying formations (Oehler and Sternberg, 1984; Meju, 2002) that further compound the degree of heterogeneity of the overburden in marine environments. It is practically expedient to resolve some of these issues. It is clear that most of them demand a rigorous 3D modeling approach.

Previous studies examine the 3D CSEM responses of idealized deep- and shallow-water exploration targets, although there is no detailed emphasis on the problems mentioned above (e.g., Everett, 2006; Hoversten et al., 2006; Um and Alumbaugh, 2007). Here, we develop a full 3D forward modeling code and use it to investigate the issues highlighted above so as to complement the past $3 \mathrm{D}$ simulation studies. In particular, we study in detail the effect of near-surface 3D heterogeneities on the 3D CSEM response of shallow-water and deepwater reservoirs, which to our knowledge has not been investigated. The main thrust is to provide a practical understanding of most of the above exploration challenges, and for each case attempt to provide a way forward in solving the attendant problem. The effect of formation anisotropy (e.g., Everett and Constable, 1999; Lu and Xia, 2007) is not considered here. For simplicity, we define deep water as deeper than $300 \mathrm{~m}$ and shallow water as $300 \mathrm{~m}$ deep or less.

\section{D MODEL STUDY AND IMPLICATIONS}

We have developed an efficient 3D staggered-grid finite-difference (FD) modeling code that enables us to accurately simulate the

a)

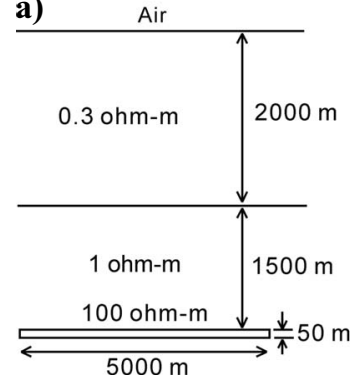

Figure 1. Three-dimensional hydrocarbon reservoir models with two seawater depths. (a) Deepwater case. (b) Shallow-water case. The horizontal and vertical dimensions of the reservoir are $5 \mathrm{~km}$ $\times 5 \mathrm{~km} \times 50 \mathrm{~m}$. marine CSEM responses of 3D reservoir targets. The methodology is described in Appendix A. Here, we first check the accuracy of our FD solutions by comparison with 1D semianalytic solutions, and then we present the results of our FD modeling to infer some useful characteristics of the 3D marine CSEM response.

For the reservoir simulation study, we consider two 3D models shown in Figure 1. Both models consist of a resistive hydrocarbon reservoir embedded in a homogeneous background sediment with a resistivity of $1 \mathrm{ohm}-\mathrm{m}$. The reservoir has a resistivity of $100 \mathrm{ohm}-$ $\mathrm{m}$ and a size of $5 \mathrm{~km} \times 5 \mathrm{~km} \times 50 \mathrm{~m}$ along the $x$-, $y$-, and $z$-coordinates, respectively. Its upper boundary is located at a depth of 1500 $\mathrm{m}$ below the seafloor. The seawater resistivity is $0.3 \mathrm{ohm}-\mathrm{m}$. The only difference between the two models is the water depth, which is either $2000 \mathrm{~m}$ or $300 \mathrm{~m}$. A single profile with 43 inline horizontal electric dipole transmitters is assumed to run through the center of the reservoir $(x, y=0 \mathrm{~km})$ along the $x$-coordinate. Each source has a length of $200 \mathrm{~m}$ and is positioned $30 \mathrm{~m}$ above the seafloor. The receivers are positioned every $200 \mathrm{~m}$ on the seafloor at $x=-8.5$ to 8.5 $\mathrm{km}$. Unlike past simulation studies, we also investigate the distorting influence of near-surface heterogeneities on the CSEM responses of a 3D target in shallow-water and deepwater situations.

\section{Electric field response}

To provide a self-check on the FD solution, we first calculated the responses of a 1D model having a 100-ohm-m layer in place of the $3 \mathrm{D}$ reservoir for the deepwater model (Figure 1a). The grid that was used has $189 \times 78 \times 52$ cells with a minimum cell size of $100 \times 100$ $\times 50 \mathrm{~m}$ in the anomalous region and a maximum size of $3.2 \times 3.2$ $\times 3.2 \mathrm{~km}$ in the corner of the boundaries. The source frequency is $0.25 \mathrm{~Hz}$. The comparison between the FD and semianalytic 1D solutions is shown in Figure 2, wherein the amplitude and phase of the inline electric fields are plotted as a function of source-receiver offset. The agreement is excellent in both the amplitude and phase responses.

As an additional self-check in our 3D reservoir investigation, we calculated the responses of the deepwater model using two grids. One grid is the same as the one used in the previous check. The other grid consists of $103 \times 48 \times 52$ cells, with a minimum cell size of 200 $\times 200 \times 50 \mathrm{~m}$ and a maximum size $6.4 \times 6.4 \times 3.2 \mathrm{~km}$. The source frequencies are 0.25 and $0.75 \mathrm{~Hz}$. In Figure 3, the amplitude and phase of the inline electric fields at selected source-receiver separations are plotted as a function of source-receiver midpoint, yielding what might be termed apparent common-depth-point (CDP) profiles. This type of data representation provides a convenient way of roughly identifying the lateral extent of the target response (Ellingsrud et al., 2002; Darnet et al., 2007; Johansen, et al., 2007). Although for each plot two values are calculated for the transmitter located on the left and right sides of the receiver, we show only the responses for the transmitter on the left because the two sets of profiles are indistinguishable.

When the coarser grid was used, all the iterative solutions at both frequencies converged to a prescribed threshold level of $10^{-6}$. For the finer grid, the solutions reaching the threshold level are for 17 and 42 source locations (out of 43 locations) at frequencies of 0.25 and $0.75 \mathrm{~Hz}$, respectively; the largest residual norm was $0.5 \times 10^{-4}$
Figure 2. A comparison of the FD (circles) and semianalytic (solid lines) solutions for a $1 \mathrm{D}$ model having a $100-\mathrm{ohm}-\mathrm{m}$ layer in place of the 3D reservoir in the deepwater model (Figure 1a) for a frequency of $0.25 \mathrm{~Hz}$. The (a) amplitude and (b) phase of the inline horizontal electric field are shown. The dashed lines show the background responses. 
and occurred when the source is located at a distance away from the reservoir region. Despite the fact that the convergence rate is relatively poor for the finer grid at $0.25 \mathrm{~Hz}$, the comparison between the two discretizations is good, with a maximum relative difference in amplitude being about $3.2 \%$ and a maximum phase difference being $0.9^{\circ}$. The average computation time per one source location and one frequency was approximately $48 \mathrm{~s}$ for the coarse grid and $293 \mathrm{~s}$ for the fine grid on a personal computer (PC) with a $2.50-\mathrm{GHz}$ Core 2 processor. All calculations in the following investigation of 3D reservoir response characteristics use the fine grid.

In Figure 3, the effect of reservoir is evident in amplitude and phase at both frequencies. Because the electromagnetic (EM) field decays more rapidly as the frequency increases, the Tx-Rx offset range in which the effect of reservoir is recognizable becomes shorter at $0.75 \mathrm{~Hz}$, if the noise floor for the electric field measurement is assumed to be at $10^{-15} \mathrm{~V} /\left(\mathrm{Am}^{2}\right)$

The electric field responses for the shallowwater model (Figure 1b) are shown in Figure 4. Because the effect of reservoir is only slightly visible at $0.75 \mathrm{~Hz}$ for this model, we present the responses at 0.25 and $0.1 \mathrm{~Hz}$ that give target signatures more clearly. It is well known that if CSEM data are acquired in shallow water, because of the interfering effect of the airwave, the resistive target response is significantly smaller in amplitude than that in the deepwater case, as shown in Figure 4a and c. It has been recognized also that phase response provides information that is crucial for identifying resistive objects (Mittet, 2008). This is illustrated in Figure $4 \mathrm{~b}$ and $\mathrm{d}$, wherein phase anomalies can be seen clearly at different Tx-Rx offsets depending on the frequency. However, the magnitude of the phase shift is small compared to the deepwater case, and the presence of a resistive reservoir generally moves the phase curves downward, which is in the opposite direction to the deepwater case.

Similar qualitative behavior can be seen in the corresponding 1D response shown in Figure 5, wherein the amplitude and phase are plotted against Tx-Rx offset at frequencies of 0.75, 0.25, and $0.1 \mathrm{~Hz}$ for the background and 1D reservoir models. Note that the lower the frequency, the longer the Tx-Rx offset at which the airwave becomes dominant (e.g., Um and Alumbaugh, 2007; Andreis and MacGregor, 2008). Comparisons between $3 \mathrm{D}$ and $1 \mathrm{D}$ responses are discussed in more detail later.

\section{Normalized electric field response}

It has been a common practice to normalize the electric field response by the response of a reference model so as to highlight the target response (e.g., Constable and Srnka, 2007). Figures 6 and 7 show the electric field responses normalized by those of the background half-space (with the wa- ter layer) for the deep- and shallow-water cases, respectively. It is important to note that whereas such a simple normalization of the data by the half-space response is useful for mitigating the effect of the source-receiver geometry, it is generally not practicable for identifying the target response for the real subsurface, which contains 3D resistivity variations.

In Figure 6, we see that after the normalization, the effect of reservoir is represented much more clearly. The peak in the normalized
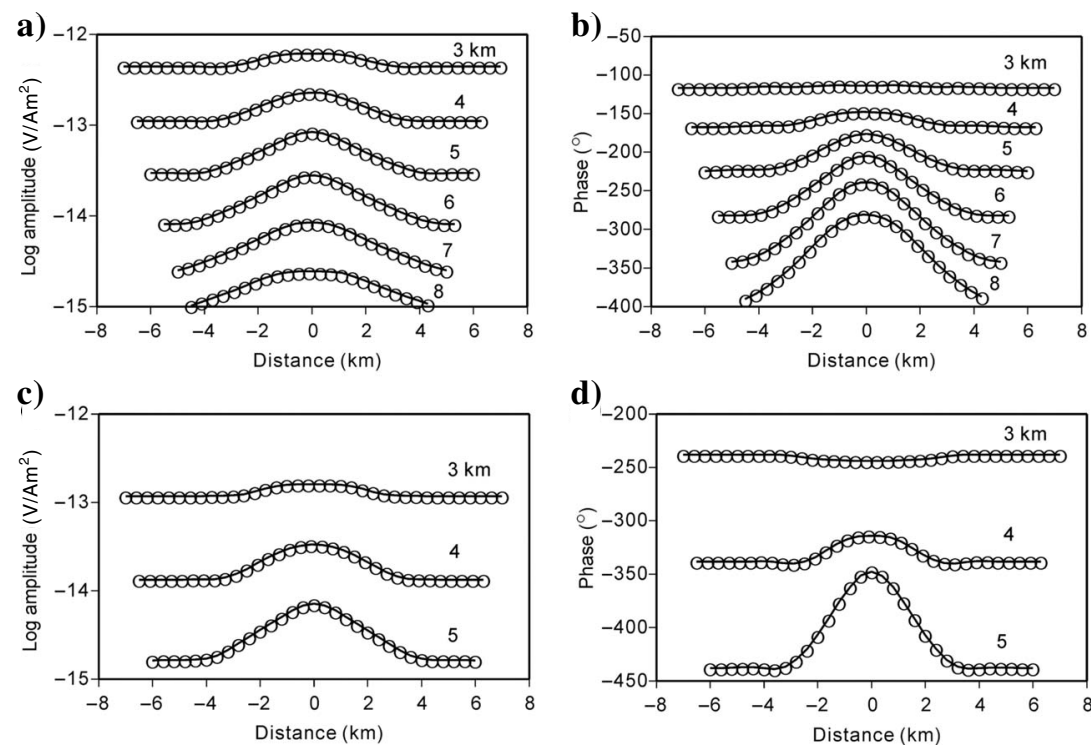

d)

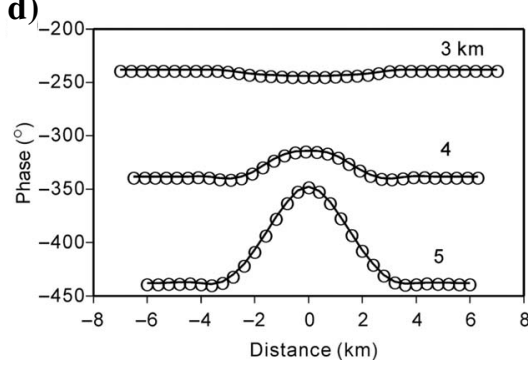

Figure 3. The amplitudes and phases of the inline horizontal electric fields at selected offsets, plotted as a function of the source-receiver midpoint for the model shown in Figure 1a (deepwater case). (a) Amplitude at $0.25 \mathrm{~Hz}$. (b) Phase at $0.25 \mathrm{~Hz}$. (c) Amplitude at $0.75 \mathrm{~Hz}$. (d) Phase at $0.75 \mathrm{~Hz}$. The solid lines and circles show the responses calculated using the fine and coarse grids, respectively.

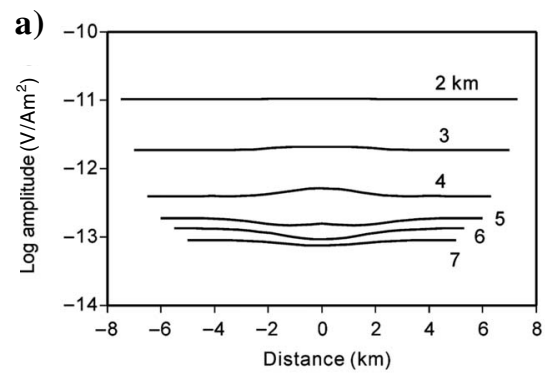

c)

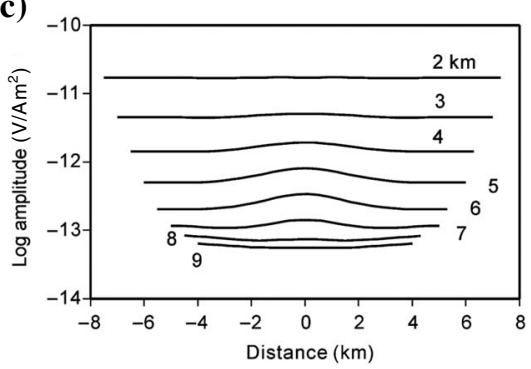

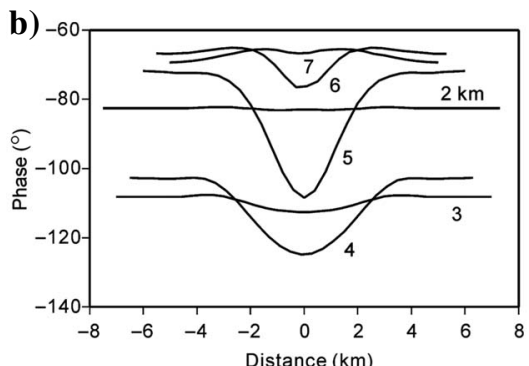

d)

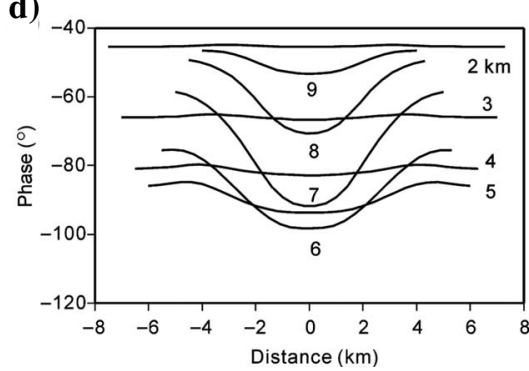

Figure 4. The amplitudes and phases of the inline horizontal electric fields at selected offsets, plotted as a function of the source-receiver midpoint for the model shown in Figure $1 \mathrm{~b}$ (shallow-water case). (a) Amplitude at $0.25 \mathrm{~Hz}$. (b) Phase at $0.25 \mathrm{~Hz}$. (c) Amplitude at $0.1 \mathrm{~Hz}$. (d) Phase at $0.1 \mathrm{~Hz}$. 
Figure 5. The amplitudes (left column) and phases (right column) of the inline horizontal electric fields at $0.75,0.25$, and $0.1 \mathrm{~Hz}$ as a function of the source-receiver separation for the 1D reservoir and background models. The thin lines are for the background model, and the thick lines are for the 1D reservoir model. For the 1D reservoir model, the 3D reservoir in Figure 1b (shallow-water case) is replaced by an infinite layer.

Figure 6. The normalized amplitudes and phase shifts for the electric field responses in Figure 3 (deepwater case). (a) Normalized amplitude at $0.25 \mathrm{~Hz}$. (b) Phase shift at $0.25 \mathrm{~Hz}$. (c) Normalized amplitude at $0.75 \mathrm{~Hz}$. (d) Phase shift at $0.75 \mathrm{~Hz}$.
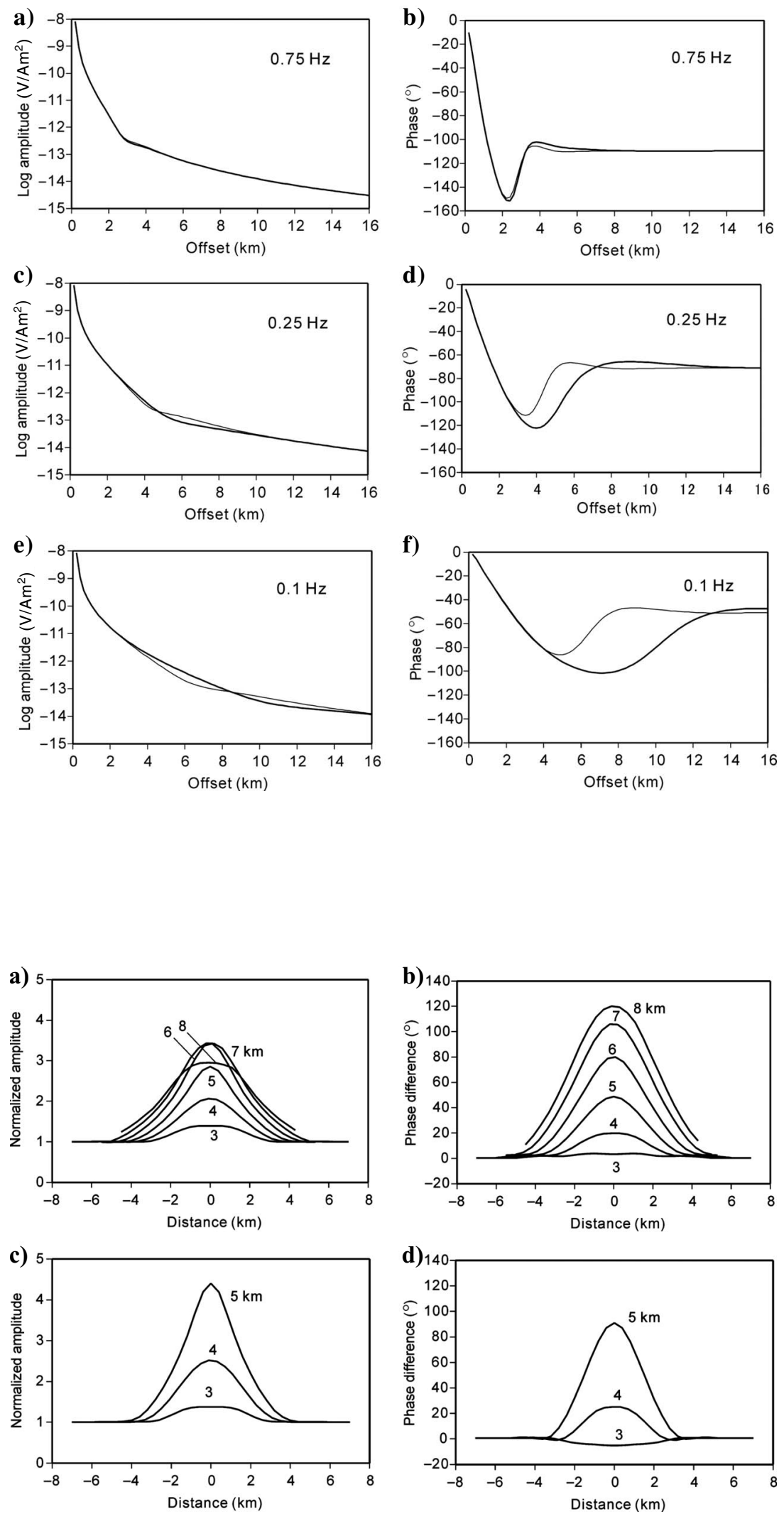
amplitude reaches a value of about 3.4 at $7-\mathrm{km}$ offset for $0.25 \mathrm{~Hz}$, and about 4.4 at $5-\mathrm{km}$ offset for $0.75 \mathrm{~Hz}$. The phase shift increases with increasing offset and attains a value of about $120^{\circ}$ at $0.25 \mathrm{~Hz}$ and $91^{\circ}$ at $0.75 \mathrm{~Hz}$. It is clear that for the deepwater case, the target signature is sufficiently large to detect and interpret reliably in both amplitude and phase at 0.25 and $0.75 \mathrm{~Hz}$.

Figure $7 \mathrm{a}$ and $\mathrm{c}$ shows that in shallow water, the effect of a reservoir on the amplitude response is to increase it slightly at intermediate offsets and decrease it at long offsets. Similar behavior can be seen in the corresponding 1D response (Figure 5). The decrease at longer offsets probably results from some coupling between the reservoir and the airwave - which is represented in $1 \mathrm{D}$ by an infinite sum of reflections off the seabed and sea surface (e.g., Chave and Cox, 1982). It is possible that the interaction of the fields diffusing upward from the reservoir (with some absolute phase) and the fields diffusing downward from the air (with some other absolute phase) has changed in the shallow water, canceling out some phase and leading to amplitude decrease. The normalized amplitude at $0.25 \mathrm{~Hz}$ lies in a range between 0.7 and 1.3 , and the peak in the normalized amplitude at $0.1 \mathrm{~Hz}$ reaches a value of about 1.7. Such target signatures might be detectable in favorable environments.

Figure $7 \mathrm{~b}$ and $\mathrm{d}$ shows that the maximum phase shift is $37^{\circ}$ (at $5-\mathrm{km}$ offset) at $0.25 \mathrm{~Hz}$ and $34^{\circ}$ (at $7-\mathrm{km}$ offset) at $0.1 \mathrm{~Hz}$, which also are detectable if accurate absolute phase measurements are made (Mittet, 2008). The maximum phase shift at $0.1 \mathrm{~Hz}$ is slightly smaller than that at $0.25 \mathrm{~Hz}$. This is in contrast to the 1D situation, where the target signature becomes larger consistently in both amplitude and phase for some frequency range as the frequency decreases (Figure 5). In addition, the phase response has sharper edges at $0.25 \mathrm{~Hz}$ than at $0.1 \mathrm{~Hz}$, which implies that the $0.25-\mathrm{Hz}$ data provide better resolution. This modeling example demonstrates that, as noted by Andreis and MacGregor (2008), it is more important in shallow water to optimize the frequencies for a given target.

\section{Effect of shallow inhomogeneities}

To simulate the data distortion arising from 3D near-surface heterogeneities such as gas pockets and elongate patches of geochemical alteration halo in the overburden of leaky fields (Oehler and Sternberg, 1984; Meju, 2002), we consider two types of anomalous shallow 3D bodies to be present in addition to the previous reservoir models as shown in Figure 8. The first type of anomalous bodies consists of seven small-scale bodies with a size of $500 \times 500 \times 100 \mathrm{~m}$ and a burial depth of $100 \mathrm{~m}$ below the seafloor. The second type consists of an extensive, elongate body of size $4000 \times 2000 \times 100 \mathrm{~m}$ and burial depth of $200 \mathrm{~m}$. The resistivity of these anomalous bodies is fixed at $20 \mathrm{ohm}-\mathrm{m}$.

Note that the anomalous bodies are located only on the left side of the center of the reservoir. These models represent two extreme cases in which near-surface 3D heterogeneities complicate the interpretation of CSEM data in very different ways. As was the case with modeling the reservoir responses, the accuracy of the FD solutions for such 3D models was checked by comparing the responses of single near-surface bodies calculated using two different grids as well as comparisons with the corresponding 1D solutions.

First we examine the effect of small-scale near-surface heterogeneities on the reservoir responses for the deep- and shallow-water cases. In Figure 9, we show the electric field responses at 0.25 and $0.75 \mathrm{~Hz}$ for the deepwater case. It can be seen from the amplitude profiles (Figure 9a and c) that the near-surface responses (or shortwavelength components) are almost independent of the frequency and that their magnitudes are almost the same (on the logarithmic scale) regardless of the Tx-Rx offset. In contrast, the near-surface
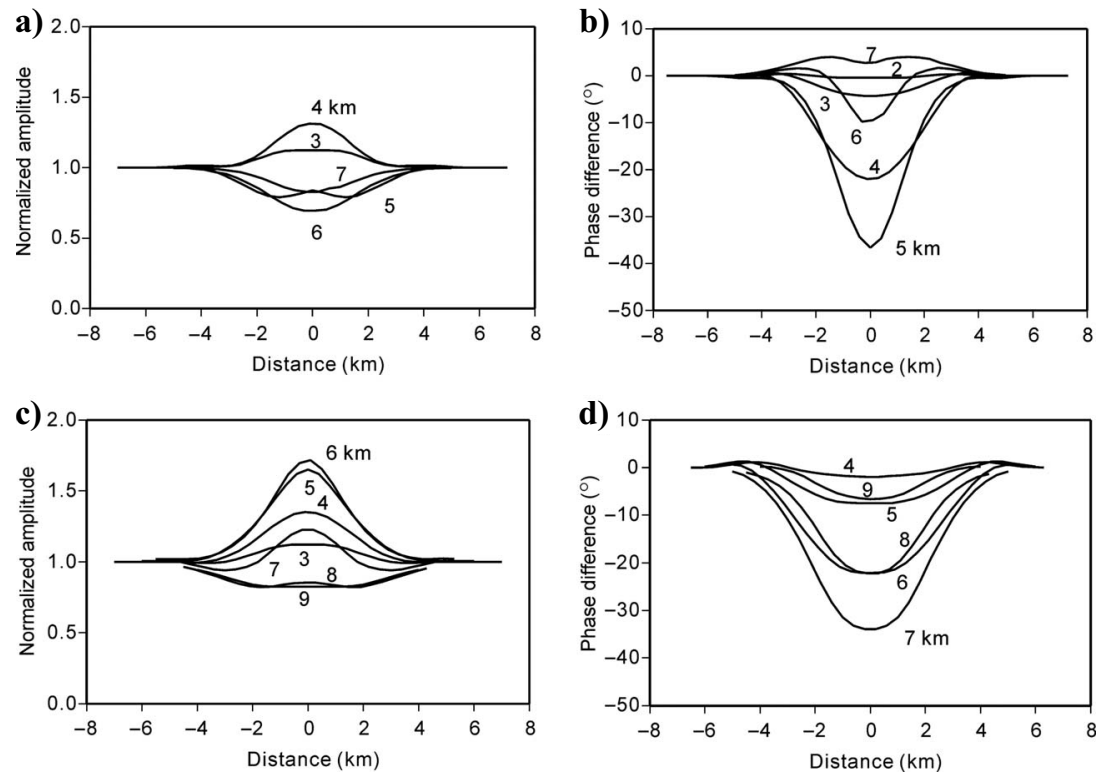

Figure 7. The normalized amplitudes and phase shifts for the electric field responses in Figure 4 (shallow-water case). (a) Normalized amplitude at $0.25 \mathrm{~Hz}$. (b) Phase shift at $0.25 \mathrm{~Hz}$. (c) Normalized amplitude at $0.1 \mathrm{~Hz}$. (d) Phase shift at $0.1 \mathrm{~Hz}$.

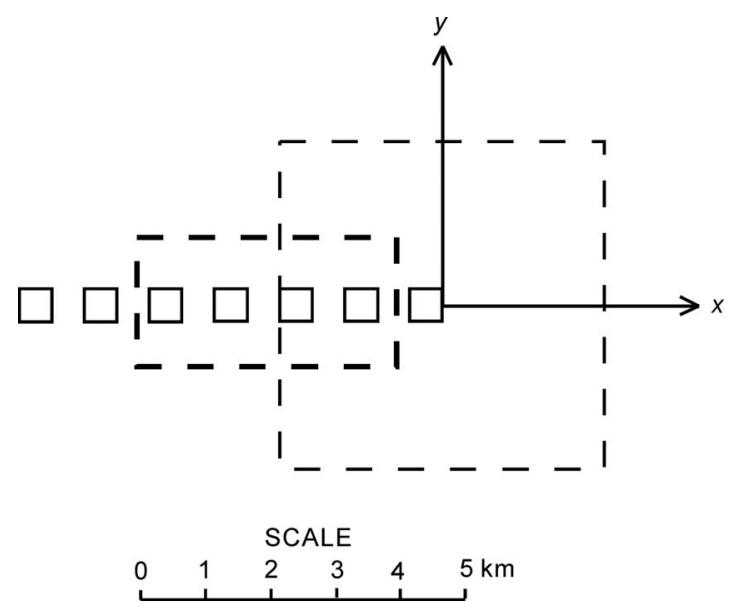

Figure 8. Plan view of two different near-surface structures shown relative to the target reservoir. The seven small squares (solid line) represent small-scale resistivity variations, and the rectangle (thick dashed line) represents a large-scale structure. All of them are $100 \mathrm{~m}$ thick. The burial depth is $100 \mathrm{~m}$ for the small-scale structures and $200 \mathrm{~m}$ for the large-scale structure. The survey line is located over the centers of these bodies and the reservoir (along the $x$-coordinate). 
phase response decreases as the offset increases and becomes almost invisible at offsets of more than about $6 \mathrm{~km}$. This feature indicates that the near-surface distortions are mainly the result of the galvanic effect, which is caused by the electric charge buildup at the boundaries of resistivity anomalies (LeMouel and Menvielle, 1982), and that the inductive effects are present also at smaller offset.

Figure 10 shows the electric field responses at 0.25 and $0.1 \mathrm{~Hz}$ for the shallow-water case. The amplitude distortions are independent of the frequency again, and unlike in the deepwater case, the phase distortions persist at all offsets. This means that the inductive effects persist at all offsets. Whereas the maximum amplitude distortion (i.e., the ratio of the amplitude response of the reservoir plus nearsurface structure to that of the reservoir model) is 1.3 in the deep water, it is only 1.1 in the shallow water. The maximum phase difference created by the near-surface structure is about $3^{\circ}$ for the shallowwater model, as opposed to $13^{\circ}$ at $0.25 \mathrm{~Hz}$ and $25^{\circ}$ at $0.75 \mathrm{~Hz}$ for the deepwater model. Such distortions are not significant, and therefore the reservoir signatures still can be seen on both the amplitude and phase profiles in shallow water.

Next we illustrate the effect of an extensive near-surface anomaly on the reservoir responses. Figure 11 shows the electric field responses of the deepwater model at 0.25 and $0.75 \mathrm{~Hz}$. Unlike the small-scale near-surface resistivity variations, the elongate resistive body generates pronounced near-surface amplitude responses, which overwhelm the target responses at smaller offsets. However, the near-surface distortions get smaller as the offset increases, and therefore the target responses are recognizable at longer offsets for both 0.25 and $0.75 \mathrm{~Hz}$. The effect of the extensive near-surface structure is largest when the source and receiver are over the structure, and diminishes when one or both are off the structure.

Note that when the reservoir signatures are peaked at a distance of $x=0 \mathrm{~km}$, the near-surface signatures are peaked at $x=-2.7 \mathrm{~km}$. The sharp breaks-in-slope in the amplitude curves appear to be caused by the galvanic effect because the distance at which the breaks appear corresponds to the position where either the source or receiver is located over the edge of the near-surface anomaly. Comparing the responses at 0.25 and $0.75 \mathrm{~Hz}$ shows that the higher frequency $(0.75 \mathrm{~Hz})$ responses are more affected by the near-surface anomaly, suggesting that broadband measurements are desirable in complex settings.

Figure 12 shows the electric field responses at 0.25 and $0.1 \mathrm{~Hz}$ for the shallow-water case. As in the deepwater case, the near-surface distortions almost dominate the response at shorter offsets. However, it is encouraging to see again that the near-surface signatures are much smaller in magnitude (on the logarithmic scale) than those in the deep water and that the reservoir signatures still are visible at longer offsets. This characteristic of near-surface responses can be viewed as a positive aspect for CSEM surveys in shallow water.

\section{Magnetic field response}

An important practical issue is how much improvement can be achieved in detecting a 3D target when both the electric and magnetic field data are used. Figure 13 shows the $y$-component of the magnetic field at $0.25 \mathrm{~Hz}$ for the deepwater model (Figure 1a), plotted in the same way as the electric field responses. Comparing these magnetic field responses to the electric ones (Figure $3 \mathrm{a}$ and b) shows that the behavior of the target signatures in the two field responses is similar in amplitude and phase, and that their magnitude is compara-
Figure 10. The amplitudes and phases of the inline horizontal electric fields at selected offsets for the shallow-water model with small-scale resistive bodies. (a) Amplitude at $0.25 \mathrm{~Hz}$. (b) Phase at $0.25 \mathrm{~Hz}$. (c) Amplitude at $0.1 \mathrm{~Hz}$. (d) Phase at $0.1 \mathrm{~Hz}$. 
ble. This is consistent with an observation from a 1D inversion study (Key, 2009), showing that separate inversions of deepwater electric and magnetic field perform equally well at recovering the reservoir and that there is no improvement in resolution from jointly inverting both fields.

Figure 14 shows the magnetic field responses of the shallow-water model (Figure 1b) at $0.25 \mathrm{~Hz}$. The target signatures in these re- sponses are quite different from those in the electric field responses (Figure $4 \mathrm{a}$ and $\mathrm{b}$ ) and are considered to be affected by the airwave in a different manner from the electric field.

The significant message here is that whether CSEM data are acquired in deep or shallow water, the magnetic field responses do provide target signatures that are nearly comparable to those in the electric field responses. In terms of response presentation, we experi-
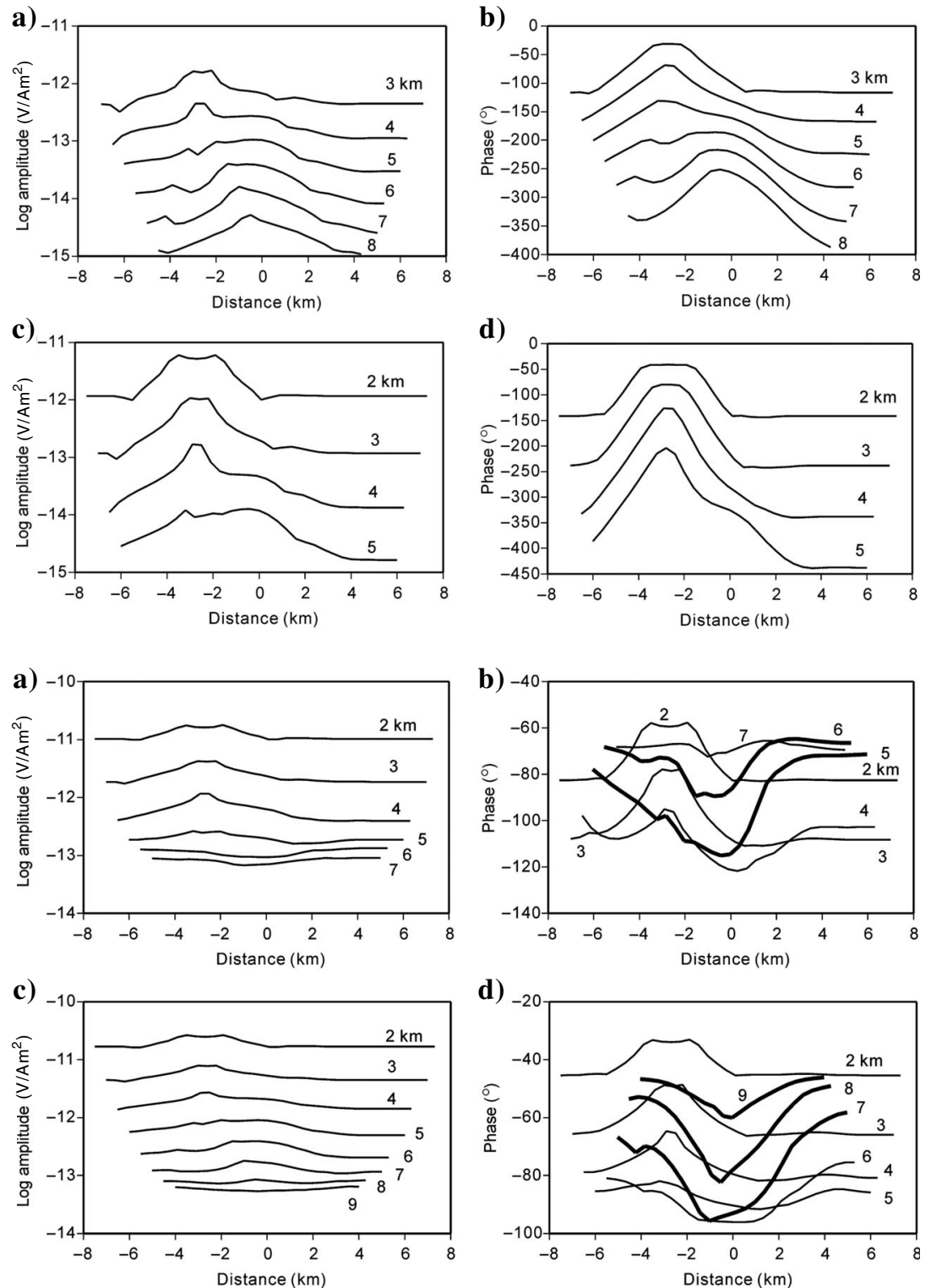

d)
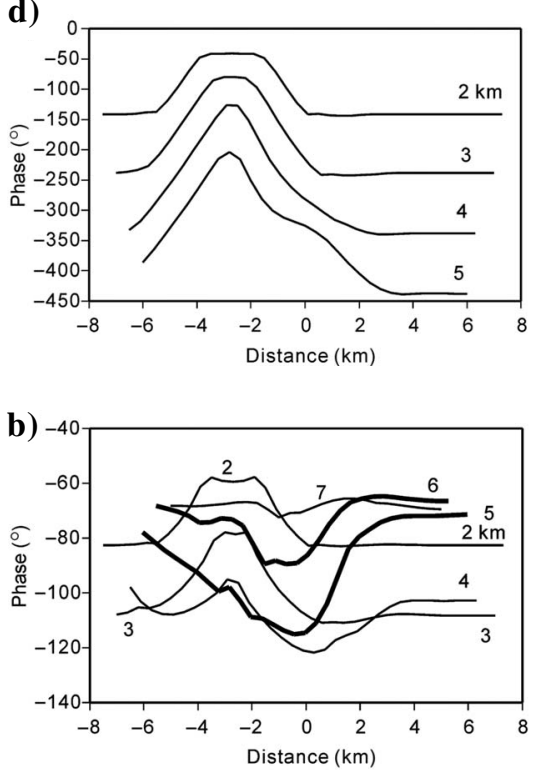

d)
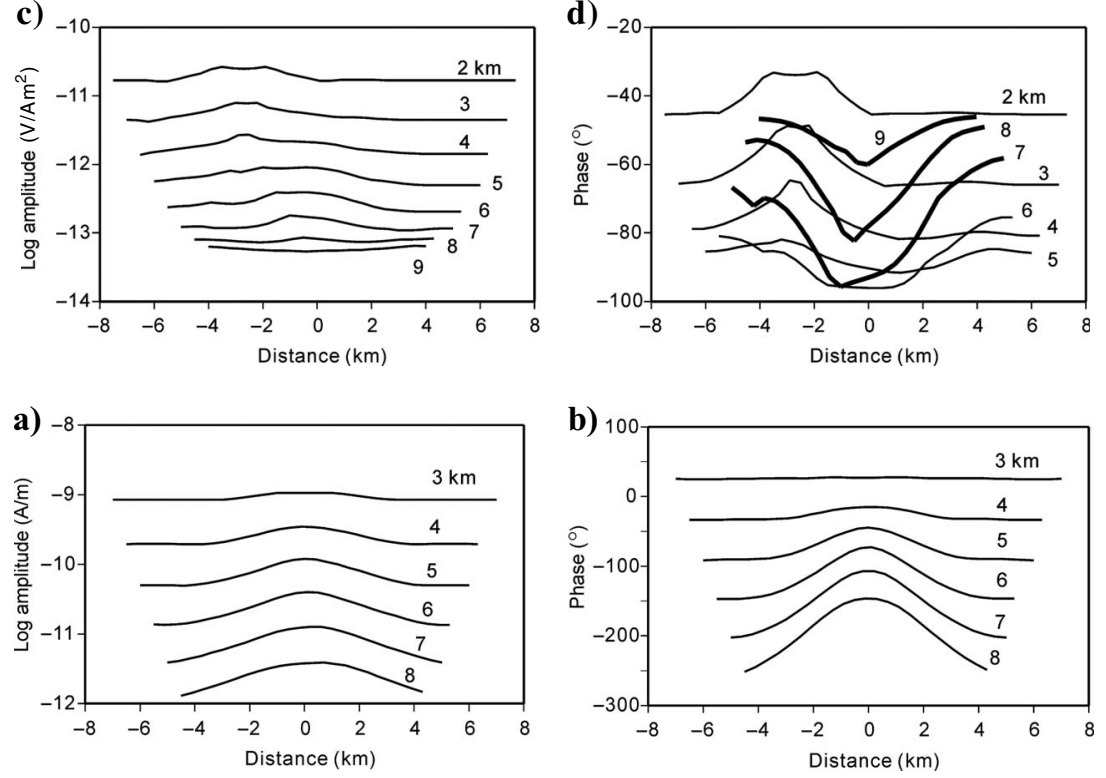

Figure 11. The amplitudes and phases of the inline horizontal electric fields at selected offsets for the deepwater model with a large-scale resistive body. (a) Amplitude at $0.25 \mathrm{~Hz}$. (b) Phase at $0.25 \mathrm{~Hz}$. (c) Amplitude at $0.75 \mathrm{~Hz}$. (d) Phase at $0.75 \mathrm{~Hz}$.

Figure 12. The amplitudes and phases of the inline horizontal electric fields at selected offsets for the shallow-water model with a large-scale resistive body. (a) Amplitude at $0.25 \mathrm{~Hz}$. (b) Phase at $0.25 \mathrm{~Hz}$. (c) Amplitude at $0.1 \mathrm{~Hz}$. (d) Phase at $0.1 \mathrm{~Hz}$.
Figure 13. The (a) amplitudes and (b) phases of the horizontal magnetic fields at selected offsets for the deepwater model (Figure 1a). The frequency is $0.25 \mathrm{~Hz}$. 

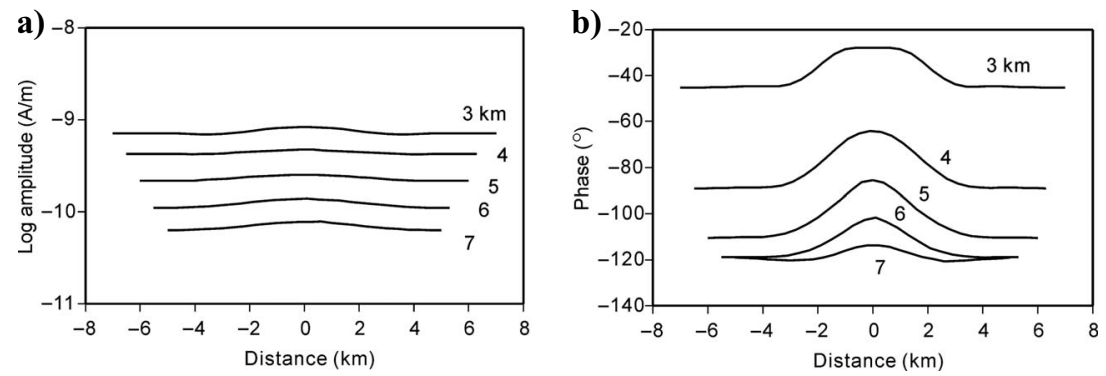

Figure 14. The (a) amplitudes and (b) phases of the horizontal magnetic fields at selected offsets for the shallow-water model (Figure 1b). The frequency is $0.25 \mathrm{~Hz}$. creases (and as the frequency decreases), and the reservoir signature still can be discerned, even in shallow water.

3) The amplitude and phase characteristics of the magnetic response for the orthogonal direction are remarkably comparable to those of the inline electric field in deep water but differ markedly in shallow water. The magnetic field responses are affected by the airwave in a different manner from the electric field in shallow water, and their joint analysis should provide a means for handling the airwave problem.

In conclusion, we opine that realistic analysis mented with the ratio of the electric to magnetic fields Ex/Hy, but this cancels out the target signature in the deepwater case because the two fields have similar target signatures. For the shallow-water case, the ratio of the fields gives a somewhat enhanced signature because the two fields behave fairly differently. It is thus obvious that there is a general need for 3D joint modeling and inversion of electric and magnetic field data, taking into account the noise characteristic in each field measurement. This will be the subject of future study aimed at developing a robust inversion scheme (e.g., Sasaki and Meju, 2006a) for marine CSEM.

\section{CONCLUSIONS}

We have highlighted some issues of present concern in marine exploration for hydrocarbons, notably, shallow-water problems, background or overburden resistivity variations, rugged seabed, thin resistors of finite extent, large source-receiver separations, and CSEM response normalization/presentation. Using simple 3D reservoir models of shallow-water and deepwater environments, we investigated the response patterns of the electric and magnetic fields for various frequencies and source-receiver separations, and the effect of near-surface heterogeneities on target signatures. The key findings of this numerical modeling study are as follows:

1) For the same idealized reservoir target, the deep- and shallowwater electric field responses are very different but are diagnostic of the 3D target reservoir. The distinct shallow-water signature consists of a peaked amplitude response at intermediate Tx-Rx offsets and a depressed amplitude response at long offsets. For electric field measurements, the phase response provides crucial information for resistive target identification in shallow water. Normalizing the CSEM response by the background response markedly enhances target detection, but it is more important in shallow water to optimize the frequencies for a given target detection.

2) Small-scale resistive bodies present in the overburden distort the electric field amplitude by almost similar amounts at all Tx$\mathrm{Rx}$ offsets independent of the measurement frequency. This distortion is caused mainly by the galvanic effect. For the phase response, this near-surface effect decreases with increasing TxRx offset in deep water but persists at all offsets in shallow water. However, the magnitude of the distortion of the phase response is much less in shallow water. Compared to small-scale resistivity variations, a large-scale near-surface resistive body distorts the electric field response more significantly. However, this distortion gets smaller in amplitude as Tx-Rx offset in- of marine CSEM data requires detailed 3D forward modeling such as developed herein. This code will form the basis for the future development of a robust CSEM data inversion scheme for marine exploration data analysis. A limitation of our present 3D CSEM forward modeling scheme is that it does not incorporate the effect of reservoir and host-rock anisotropy in the calculations, but this additional level of complexity is not required to explain the potentially useful response characteristics presented here.

\section{ACKNOWLEDGMENTS}

The authors acknowledge the constructive comments of the anonymous reviewers and, in particular, the associate editor (K. Key), which improved the clarity of the paper.

\section{APPENDIX A}

\section{D FINITE-DIFFERENCE CSEM MODELING AND DIVERGENCE CORRECTION METHOD}

To simulate the marine CSEM response of a 3D subsurface structure, we numerically solve the diffusion equation for the electric field that can be derived from Maxwell's equations. Assuming a time-harmonic dependence of $e^{i \omega t}$, the secondary electric field $\mathbf{E}_{\mathrm{s}}$ satisfies

$$
\nabla \times \nabla \times \mathbf{E}_{\mathrm{s}}+i \omega \mu_{0} \sigma \mathbf{E}_{\mathrm{s}}=-i \omega \mu_{0}\left(\sigma-\sigma_{\mathrm{p}}\right) \mathbf{E}_{\mathrm{p}},
$$

where $\sigma$ is the conductivity, $\mu_{0}$ is the magnetic permeability of free space, $\omega$ is the angular frequency, and $\mathbf{E}_{\mathrm{p}}$ is the primary electric field in a $1 \mathrm{D}$ layered structure with conductivity $\sigma_{\mathrm{p}}$. Following Newman and Alumbaugh (1995), we use a staggered grid to discretize the model region (including the air layer). As boundary conditions, the tangential component of the secondary field is set equal to zero on the boundaries of the model. Approximating equation A-1 with finite differences at a specific frequency results in a linear system of equations,

$$
\mathbf{K f}=\mathbf{s},
$$

where $\mathbf{K}$ is a symmetrical complex matrix, $\mathbf{f}$ is the unknown vector for the three components of the secondary electric field, and $\mathbf{s}$ is the source vector resulting from the right-hand side of equation A-1 and the boundary conditions. The entries of $\mathbf{K}$ are real except on the main diagonal entries, which depend on the conductivity $\sigma$. After the sec- 
ondary fields are obtained, the total fields can be calculated from

$$
\mathbf{E}=\mathbf{E}_{\mathrm{p}}+\mathbf{E}_{\mathrm{s}} .
$$

We use the biconjugate gradient (BCG) method to find the solution of equation A-2. It is well known that equation A-2 is ill conditioned at low frequencies, and that any iterative solvers are very slow or fail to converge without an appropriate preconditioning (Mackie et al., 1994; Smith, 1996). The preconditioner we use is a variant of the classical incomplete Cholesky decomposition, in which the incomplete Cholesky decomposition is applied only to the diagonal subblocks that are positive-definite (Mackie et al., 1994). Although this preconditioner does improve the convergence rate by reducing the conditioned number of the system matrix, the BCG method still is slow to converge for the system of equations used in general 3D EM modeling. This is particularly true for marine CSEM methods in which the EM fields are generated by an electric dipole source at low frequencies. Our experience has been that the convergence for the system associated with an electric dipole source generally is slower than that with a magnetic dipole source.

The system used in EM modeling generally is difficult to solve at low frequencies because conservation of current is not guaranteed in a discretized version of equation A-1 as a result of the numerical errors when the second term of the left-hand side becomes small. To overcome this inherent difficulty, we use a correction method that enforces divergence-free conditions on the current density in the earth and the electric field in the air (Smith, 1996; Sasaki, 2001). The conditions that must hold for the secondary electric field are

$$
\nabla \cdot \sigma \mathbf{E}_{\mathrm{s}}+\nabla \cdot\left(\sigma-\sigma_{\mathrm{p}}\right) \mathbf{E}_{\mathrm{p}}=0
$$

in the earth, and

$$
\nabla \cdot \mathbf{E}_{\mathrm{s}}=0
$$

in the air. Note that equation A- 4 can be obtained by taking the divergence of equation A-1. If the divergences in equations A-4 and A-5 have nonzero values denoted by $\psi_{\mathrm{e}}$ and $\psi_{\mathrm{a}}$, respectively, then we can update the solution of equation A-2 by solving

$$
\nabla \cdot \sigma \nabla \phi=-\psi_{e}
$$

and

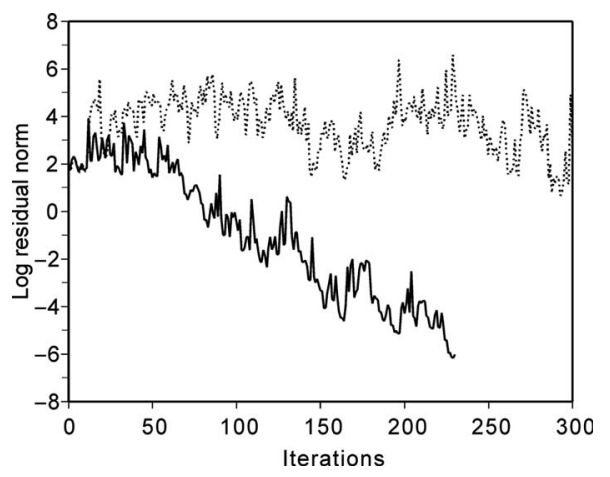

Figure A-1. A comparison of the convergence of the BCG method for the cases with (solid lines) and without (dotted lines) the divergence correction, obtained when computing the response of the deepwater model at $0.25 \mathrm{~Hz}$ for a source location $x=5.2 \mathrm{~km}$ on the fine grid (Figure $3 a$ and $b$ ).

$$
\nabla \cdot \nabla \phi=-\psi_{a}
$$

and then by adding $\nabla \phi$.

We solve equations A-6 and A-7 for real parts and imaginary parts separately using the finite-difference method in a similar way to the DC resistivity modeling (e.g., Dey and Morrison, 1979). Each scalar differential equation (Poisson's equation) only needs to be solved approximately. In the modeling examples shown before, this divergence correction is invoked every twenty BCG iterations or if the residual norm decreases by more than $50 \%$ of that obtained after the previous correction. The residual norm is defined by $\|\mathbf{K f}-\mathbf{s}\|^{2}$ normalized by $\|\mathbf{s}\|^{2}$. The BCG solution is assumed to converge to an acceptable accuracy when this residual norm is less than a prescribed tolerance.

A comparison of the convergence for the cases with and without divergence correction is shown in Figure A-1, wherein the residual norm versus iteration number is plotted for the computation at $0.25 \mathrm{~Hz}$ on the finer grid for a source location $(x=5.2 \mathrm{~km})$ in Figure $3 \mathrm{a}$ and $\mathrm{b}$. It is evident that the convergence of the iterative solution is improved significantly by using the divergence correction.

\section{REFERENCES}

Andreis, D., and L. MacGregor, 2008, Controlled-source electromagnetic sounding in shallow water: Principles and applications: Geophysics, 73, no. 1, F21-F32.

Chandola, S. K., R. Karim, A. Mawarni, R. Ismail, N. Shahud, R. Rahman, P. Bernabe, and K. Brauti, 2007, Challenges in shallow water CSEM surveying: A case history from Southeast Asia: International Petroleum Technology Conference, Extended Abstract, Paper No. IPTC-11511-PP.

Chave, A. D., and C. S. Cox, 1982, Controlled electromagnetic sources for measuring electrical conductivity beneath the oceans: 1 - Forward problem and model study: Journal of Geophysical Research, 87, 5327-5338.

Constable, S., and L. J. Srnka, 2007, An introduction to marine controlledsource electromagnetic methods for hydrocarbon exploration: Geophysics, 72 , no. 2 , WA3-WA 12 .

Constable, S., and C. J. Weiss, 2006, Mapping thin resistors and hydrocarbons with marine EM methods: Insights from 1D modeling: Geophysics, 71, no. 2, G43-G51.

Darnet, M., M. C. K. Choo, R.-E. Plessix, M. L. Rosenquist, K. Yip-Cheong, E. Sims, and J. W. K. Voon, 2007, Detecting hydrocarbon reservoirs from CSEM data in complex settings: Application to deepwater Sabah, Malaysia: Geophysics, 72, no. 2, WA97-WA103.

Dey, A., and H. F. Morrison, 1979, Resistivity modeling for arbitrarily shaped three-dimensional structures: Geophysics, 44, 753-780.

Eidesmo, T., S. Ellingsrud, L. M. MacGregor, S. Constable, M. C. Sinha, S. Johansen, F. N. Kong, and H. Westerdahl, 2002, Seabed logging (SBL), a new method for remote and direct identification of hydrocarbon filled layers in deepwater areas: First Break, 20, 144-152.

Ellingsrud, S., T. Eidesmo, S. Johansen, M. C. Sinha, L. M. MacGregor, and S. Constable, 2002, Remote sensing of hydrocarbon layers by seabed logging (SBL): Results from a cruise offshore Angola: The Leading Edge, 21, 972-982.

Everett, M. E., 2006, Finite element analysis of shallow-water marine controlled-source electromagnetic signals for hydrocarbon exploration: Presented at the 3rd International Marine Electromagnetics Conference (MARELEC).

Everett, M. E., and S. Constable, 1999, Electric dipole fields over an anisotropic seafloor: Theory and application to the structure of $40 \mathrm{Ma}$ Pacific Ocean lithosphere: Geophysical Journal International, 136, 41-56.

Hoversten, G. M., G. A. Newman, N. Geier, and G. Flanagan, 2006, 3D modeling of a deepwater EM exploration survey: Geophysics, 71, no. 5, G239G248.

Johansen, S. E., T. A. Wicklund, and H. E. F. Amundssen, 2007, Interpretation example of marine CSEM data: The Leading Edge, 26, 348-354.

Key, K., 2009, 1D inversion of multicomponent, multifrequency marine CSEM data: Methodology and synthetic studies for resolving thin resistive layers: Geophysics, 74, no. 2, F9-F20.

LeMouel, J. L., and M. Menvielle, 1982, Geomagnetic variation anomalies and deflection of telluric currents: Geophysical Journal of the Royal Astronomical Society, $68,575-587$.

Li, Y., and S. Constable, 2007, 2D marine controlled-source electromagnetic 
modeling: Part 2 - The effect of bathymetry: Geophysics, 72, no. 2, WA63-WA71.

Lu, X., and C. Xia, 2007, Understanding anisotropy in marine CSEM data: 77th Annual International Meeting, SEG, Expanded Abstracts, 633-637.

Mackie, R. L., J. T. Smith, and T. R. Madden, 1994, Three-dimensional electromagnetic modeling using finite difference equations: The magnetotelluric example: Radio Science, 29, 923-935.

Meju, M. A., 2002, Geoelectromagnetic exploration for natural resources: Models, case studies and challenges: Surveys in Geophysics, 23, 133-205.

Mittet, R., 2008, Normalized amplitude ratios for frequency-domain CSEM in very shallow water: First Break, 26, 47-54.

Newman, G. A., and D. L. Alumbaugh, 1995, Frequency-domain modeling of airborne electromagnetic responses using staggered finite differences: Geophysical Prospecting, 43, 1021-1042.

Oehler, D. Z, and B. K. Sternberg, 1984, Seepage-induced anomalies, "false" anomalies, and implications for electrical prospecting: AAPG Bulletin, 68, 1121-1145.

Orange, A., K. Key, and S. Constable, 2009, The feasibility of reservoir monitoring using time-lapse marine CSEM: Geophysics, 74, no. 2, F21-F29.
Sasaki, Y., 2001, Full 3-D inversion of electromagnetic data on PC: Journal of Applied Geophysics, 46, 45-54.

Sasaki, Y., and M. A. Meju, 2006a, A multidimensional horizontal-loop controlled-source electromagnetic inversion method and its use to characterize aquiferous fractured crystalline rocks: Geophysical Journal International, 166, 59-66.

_ 2006b, Three-dimensional joint inversion for magnetotelluric resistivity and static shift distributions in complex media: Journal of Geophysical Research, 111, B05101, doi: 10.1029/2005JB004009.

Singer, B. S., E. B. Fainberg, P. O. Barsukov, and J. K. Kjerstad, 2007, Static shift in marine electromagnetic measurements: 69th Annual Conference and Exhibition, EAGE, Extended Abstracts, D033.

Smith, J. T., 1996, Conservative modeling of 3-D electromagnetic fields: Part 2 - Biconjugate gradient solution and an accelerator: Geophysics, 61 , 1319-1324.

Um, E. S., and D. L. Alumbaugh, 2007, On the physics of the marine controlled-source electromagnetic method: Geophysics, 72, no. 2, WA13WA26. 